\title{
Cartografía digital de una colección de música folclórica: «Alan Lomax's journey across Spain (1952-53)»
}

\author{
Digital Cartography of a Folk Music Collection: \\ «Alan Lomax's journey across Spain (1952-53)»
}

\author{
Ascensión Mazuela-Anguita \\ Universidad de Granada \\ https://orcid.org/0000-0002-7144-1335 \\ amazuela@ugr.es
}

Recibido: 21/07/2021; Revisado: 08/12/2021; Aceptado: 09/12/2021

\section{Resumen}

Este artículo presenta los resultados digitales de un proyecto desarrollado en 2017 en la Library of Congress (Washington D. C.) cuyo objetivo fue analizar la colección de grabaciones de música folclórica que recopiló Alan Lomax (1915-2002) en España entre junio de 1952 y enero de 1953 y los centenares de documentos resultantes de su viaje. La información completa de esta colección se incorporó a la base de datos Fondo de Música Tradicional IMF-CSIC (http:/ / musicatradicional.eu/lomax), y se generó la aplicación «Alan Lomax's journey across Spain (1952-53)» (http://arcg.is/2x1K6u0), basada en un mapa interactivo que contiene el itinerario de Lomax.

Palabras clave: Alan Lomax, música folclórica, grabación sonora, SIG, Drupal.

\begin{abstract}
This article presents the digital results of a project conducted at the Library of Congress in Washington DC in 2017. The aim of this project was to analyse the collection of folk music compiled by Alan Lomax (1915-2002) in Spain between June 1952 and January 1953, and the hundreds of documents deriving from his journey. All the data provided by the Lomax collection was incorporated into the Fondo de Música Tradicional IMF-CSIC (http://musicatradicional.eu/lomax), and the application "Alan Lomax's journey across Spain (1952-53)» (http:/ / arcg.is/2x1K6u0), which display Lomax's itinerary on an open-access interactive map, was also created.
\end{abstract}

Keywords: Alan Lomax, Spanish Folk Music, Sound Recording, GIS, Drupal. 


\section{INTRODUCCIÓN}

La carrera del folclorista norteamericano Alan Lomax (1915-2002) se inició en 1933 cuando su padre John Lomax (1867-1948) fue nombrado jefe del Archive of American Folk Song de la Library of Congress en Washington D. C. (Estados Unidos) y le solicitó que ampliara la colección de manuscritos y cilindros fonográficos del archivo a través de la realización de nuevas grabaciones en una diversidad de lugares de Estados Unidos (SzwED, 2010). En 1937, Alan Lomax fue nombrado «Assistant in Charge» del archivo y continuó llevando a cabo grabaciones de campo para la institución hasta 1942, convirtiéndose en un pionero en la grabación de historias orales de músicos americanos (LomAX, 2011). En 1942 pasó a trabajar para la Office of War Information y el Armed Forces Radio Service, produciendo programas de música folclórica pero, en la década de 1950, su asociación con organizaciones de izquierda y personas de ideología comunista lo llevó a formar parte de una «lista negra» que le impedía encontrar trabajo. En parte por este motivo y también a causa de un encargo que había recibido de la discográfica neoyorquina Columbia, Lomax se mudó a Londres y pasó ocho años colaborando con la BBC y haciendo trabajo de campo en Europa, especialmente en España e Italia, pero también en Irlanda y Escocia (LomAX, 2003: 103; PiZÀ, 2006: 14; SZWED, 2010: 268-289).

El 18 de abril de 1951 Lomax, siguiendo las indicaciones de Maud Karpeless (1885-1976), colectora británica de música folclórica, colaboradora de Cecil Sharp (1859-1924) y jefa del International Folk Music Council, escribió una carta dirigida al Instituto Británico en España, en la que explicaba que la discográfica Columbia le había encomendado reunir una biblioteca mundial de música folclórica y primitiva que se titularía World Library of Folk and Primitive Music. Cada álbum se dedicaría a un país o región y contendría una hora de grabaciones que debían de ser fruto del trabajo de campo. Uno de los álbumes estaría dedicado a la península ibérica, por lo que en su carta Lomax solicitaba orientación para encontrar un folclorista español con el que trabajar en la zona y que editase o coeditase el álbum. ${ }^{1}$ El 7 de mayo de 1951 Lomax remitió una carta a Walter Starkie (1894-1976), músico y escritor irlandés residente en Madrid como representante del British Council en España, con el mismo propósito: esperaba que este lo ayudase a encontrar a una persona con experiencia en la grabación y el trabajo de campo. Explicaba que probablemente él mismo se trasladaría a España para trabajar en el álbum, puesto que suponía que allí no había ninguna persona formada en la grabación de campo como las que había encontrado en otros países. ${ }^{2}$ Starkie le indicó que los folcloristas españoles continuaban utilizando el método de transcribir a mano las melodías y textos de las canciones, aunque quizás aquellos que trabajaban en los grandes centros dispondrían de la grabadora Webster que él mismo tenía. ${ }^{3}$

Finalmente, ante la imposibilidad de encontrar un folclorista español con las características y el equipamiento que demandaba, Lomax acudió al Congreso y Certamen Internacional de Folclore celebrado en Palma (Mallorca) entre el 21 y el 28 de junio de 1952 para establecer posibles contactos. En su artículo «Saga of a Folksong Hunter» (1960), comenta su encuentro con Marius Schneider (1903-

1 Carta de Alan Lomax al British Institute en Madrid, 18 de abril de 1951, Washington D. C., Library of Congress, American Folklife Center (en adelante, AFC), 2004/004: MS 03.05.51.

2 Carta de Walter Starkie a Alan Lomax, 11 de mayo de 1951, AFC 2004/004: MS 03.05.51.

3 Carta de Walter Starkie a Alan Lomax, 16 de mayo de 1951, AFC 2004/004: MS 03.05.51. 
1982), coordinador del festival y director de la Sección de Folclore del Instituto Español de Musicología, una institución fundada en Barcelona en 1943 a iniciativa del musicólogo y sacerdote Higini Anglès (1888-1969) con el objetivo de promover el estudio de la música española. Lomax explica que cuando habló a Schneider sobre su proyecto de llevar a cabo grabaciones de música tradicional española, este le aseguró que se encargaría personalmente de que ningún musicólogo español lo ayudase, sugiriéndole incluso abandonar el país (LOMAX, 1960; reimpreso en Lomax, 2003: 181). Lomax señala que este encuentro le hizo prometerse que grabaría la música tradicional de España aunque le llevara el resto de su vida (Lomax, 1960; reimpreso en Lomax, 2003: 182).

En efecto, desde Palma inició un periplo por casi todo el país en el que registró con su grabadora Magnecord en torno a 1.500 piezas de música de tradición oral entre junio de 1952 y enero de $1953,{ }^{4}$ contando con la ayuda de una asistente, la británica Jeanette Bell (1927-2018). ${ }^{5}$ Los resultados materiales de este viaje se conservan en el American Folklife Center de la Library of Congress en Washington D. C. comisariados por Todd Harvey e incluyen cintas magnéticas con la música grabada, pero también las cajas originales de las cintas con anotaciones, fotografías principalmente de lugares y personas tomadas por Lomax con una pequeña cámara que había comprado antes de salir de Inglaterra, cuadernos de campo manuscritos, facturas, recibos y otra documentación contable, panfletos de diferentes entidades españolas, mapas anotados que Lomax utilizó en su viaje, centenares de cartas que reflejan la prolífica correspondencia que mantuvo tras el viaje con contactos e intérpretes en España, tarjetas de visita de diferentes personalidades que lo ayudaron a localizar nuevos contactos, guiones de una serie de programas de radio que preparó para la BBC y en los que se incluyeron sus grabaciones de música española, ${ }^{6}$ diarios mecanografiados redactados tanto por Alan Lomax como por su asistente Jeanette Bell, e incluso el manuscrito de parte de un libro para publicación con Oxford University Press en colaboración con Eduardo Martínez Torner (1888-1955), musicólogo asturiano exiliado en

\footnotetext{
4 Sobre las grabaciones de Alan Lomax en España, véanse Lomax (1960); COHEN (2002 y 2011b); CoHeN et al. (2004); PIZÀ (2006); y SZWED (2010: 268-276). Acerca de las grabaciones realizadas en las islas Baleares en particular, véanse PizÀ (2006); y CoHEN (2011a). Con respecto a las realizadas en Aragón, Serrano (2000); y sobre las de Castilla y León, véanse CaSado Lobato (2003); Porro Fernández, (2010); Porro Fernández et al. (2011); CASAdo Lobato y Porro Fernández (2011 y 2012).

5 Acerca de la contribución de Bell al proceso de grabación y a la configuración de la colección de música española de Alan Lomax, que hasta ahora había pasado casi totalmente desapercibida, véase Mazuela-Anguita (2021b).

6 Las grabaciones de Lomax en España se hicieron públicas por primera vez en The Folk Music of Spain, la producción del Third Programme de la BBC en la que Lomax comentó su viaje por España en dos programas emitidos el 24 y el 29 de octubre de 1953, y dedicados a las grabaciones de Andalucía y noroeste de España, respectivamente. Sobre la labor de difusión de la canción folclórica que hizo Lomax en Londres a través de la BBC en la década de 1950, véase GREGORY (2002). Lomax describía el Third Programme de la BBC como el foro cultural más influyente del mundo occidental, con una audiencia inteligente y con mínima censura; véase LomaX, 1960; reimpreso en Lomax (2003: 182). A estas emisiones siguió una serie de seis programas escritos por Eduardo Martínez Torner y traducidos por John Gavall para la BBC bajo el título Spanish Folk Music y emitidos el 19, 28, 30 de noviembre, y el 12, 17 y 26 de diciembre de 1953. El propio Lomax escribió otra serie de seis programas para la BBC titulada Reminiscences of a Folk Song Collector sobre su trabajo de campo en España e Italia. Dos de estos programas se dedicaron a España -el número 3, titulado «Spain: From Seville to the Pyrenees» y el número 4, «Spain: Vigo to San Sebastian»-; ambos se emitieron el 21 de septiembre de 1956.
} 


\section{Londres. $^{7}$}

La aproximación de Lomax al trabajo de campo era diferente a la de los folcloristas que trabajaban en España en esa época, ${ }^{8}$ y la falta de colaboración por parte de los folcloristas españoles a la que Lomax hace referencia en sus escritos lleva a preguntarse cómo consiguió tejer una red de contactos que le permitió recorrer España y completar su proyecto. Responder a estas cuestiones fue uno de los objetivos del proyecto «The Relationship Between the Alan Lomax Collection and the Collection of Spanish Traditional Music of the Institució Milà i Fontanals of the Spanish National Research Council (CSIC-IMF) in Barcelona (1944-1966)», desarrollado entre enero y septiembre de 2017 en el John W. Kluge Center de la Library of Congress gracias a la beca postdoctoral «Jon B. Lovelace Fellowship for the Study of the Alan Lomax Collection», concedida por la Library of Congress. Este proyecto ha resultado en la monografía Alan Lomax y Jeanette Bell en España (1952-1953): las grabaciones de música folclórica, que presenta un análisis de las grabaciones realizadas por Lomax en España y la documentación relacionada con su viaje, utilizando la historia oral como marco conceptual y la tecnología como herramienta (MAZuelA-Anguita, 2021b). El presente artículo se centra en el uso que se hizo de la tecnología en este proyecto y en uno de los resultados digitales del mismo, consistente en una aplicación basada en los sistemas de información geográfica que proporciona, de forma visual e interactiva, el itinerario de Lomax por España en conexión con una base de datos a la que se ha incorporado la totalidad de la información que proporciona la colección de música española de Alan Lomax.

\section{BASES METODOLÓGICAS}

Basándose en el trabajo fundamental de Donald A. Ritchie, Doing Oral History, la Oral History Association define la historia oral como «un campo de estudio y un método de compilar, preservar e interpretar las voces y memorias de personas, comunidades y participantes en eventos pasados». Considera la historia oral como «la más antigua clase de investigación histórica, precediendo a la palabra escrita», pero también como «una de las más modernas, a partir de las grabaciones en cinta en la década de 1940 y, actualmente, con el uso de las tecnologías digitales del siglo XXI». ${ }^{9}$ Un requisito imprescindible para que una grabación se considere parte de la historia oral es que incluya una entrevista o un intercambio entre entrevistador y entrevistado. ${ }^{10}$ La historia oral se ha incorporado lentamente

7 En 2004, dos años después del fallecimiento de Lomax, el American Folklife Center adquirió la «Alan Lomax Collection», que contiene todos los materiales que este había recopilado desde que dejó de trabajar en la Library of Congress en 1942.

8 Acerca del ideario de Alan Lomax acerca del folclore, véase BARON (2012).

9 «Oral history is a field of study and a method of gathering, preserving, and interpreting the voices and memories of people, communities, and participants in past events. Oral history is both the oldest type of historical inquiry, predating the written word, and one of the most modern, initiated with tape recorders in the 1940s and now using 21st-century digital technologies» (http://www.oralhistory. org/about/do-oral-history). Citado por DAvIs (2015: 256). Véanse también VANSINA (1985); y RITCHIE (2015 [1995]).

10 Ritchie (2015 [1995]: 1): «[...] oral history collects memories and personal commentaries of historical significance through recorded interviews. An oral history interview generally consists of a well-prepared interviewer questioning an interviewee and recording their exchange in audio or 
al campo musicológico, probablemente como consecuencia de las divisiones metodológicas que tradicionalmente se han producido entre musicología y etnomusicología (PERLIS, 1994: 610). Más recientemente, se ha comentado la falta de perspectiva histórica en los trabajos etnomusicológicos y se ha abogado por la inclusión de una dimensión histórica en la etnomusicología que permita explorar las intersecciones entre historia oral, biografía musical y etnomusicología histórica que emergieron en la década de 1970 (DAvIs, 2015). Por tanto, el uso de la historia oral como marco conceptual en el análisis de los documentos resultantes del viaje de Alan Lomax por España permite introducir una dimensión histórica en su investigación etnomusicológica.

El proyecto de análisis de la colección de música española de Lomax se basó desde su inicio en la utilización de la tecnología digital como herramienta para establecer conexiones entre las piezas musicales grabadas por Lomax y otras colecciones de música tradicional española compiladas en la misma época, como la conservada en la Institución Milá y Fontanals de Investigación en Humanidades (IMF) del Consejo Superior de Investigaciones Científicas (CSIC) en Barcelona, institución heredera de los fondos del antiguo Instituto Español de Musicología. Esta colección de Barcelona incluye más de 20.000 piezas copiadas en papel y recogidas por toda España entre 1944 y 1960, la mayoría a través de las llamadas "misiones folclóricas» y los concursos que organizó el Instituto Español de Musicología. ${ }^{11}$ Desde 2011 he participado en el proyecto, dirigido por Emilio RosFábregas, para digitalizar y catalogar esta colección en una base de datos online y de libre acceso llamada Fondo de Música Tradicional IMF-CSIC (en adelante, FMT). ${ }^{12}$

En un primer estadio de mi investigación en la Library of Congress, incorporé a la misma base de datos las grabaciones realizadas por Alan Lomax en España, los nombres y datos de quienes interpretaron música para él, las fotografías que tomó, las notas de sus cuadernos de campo, diarios y escritos, sus mapas anotados y correspondencia y los lugares que visitó. Esta catalogación preparó los materiales para análisis y comparación y permitió establecer relaciones entre las grabaciones de Lomax y otros materiales de música tradicional recopilados en España en la misma época, poniendo así en contexto el trabajo de Lomax y permitiendo el establecimiento de análisis comparativos, cambios y pervivencias de las músicas españolas de tradición oral (véanse MAZUELA-ANGUITA, 2021a y 2021b). Además, este procesamiento de la información condujo a establecer una

video format. Recordings of the interview are transcribed, summarized, or indexed and then placed in a library or archives. These interviews may be used for research or excerpted in a publication, radio or video documentary, museum exhibition, dramatization or other form of public presentation. Recordings, transcripts, catalogs, photographs and related documentary materials can also be posted on the Internet. Oral history does not include random taping, such as President Richard Nixon's surreptitious recording of his White House conversations, nor does it refer to recorded speeches, wiretapping, personal diaries on tape, or other sound recordings that lack the dialogue between interviewer and interviewee».

11 Sobre la colección preservada en la IMF, véanse CALVo (1989 y 1989-1990); MARTí (1997); PELINSKI, (1997); Gembero-Ustárroz (2011); Mazuela-Anguita (2015 y 2016); y Gembero-Ustárroz y Ros-FábreGas (2021).

12 Fondo de Música Tradicional IMF-CSIC. E. Ros-Fábregas (ed.). Consejo Superior de Investigaciones Científicas, Institució Milà i Fontanals, Barcelona. [ISSN: 2564-8500]. Esta base de datos es de acceso libre en https://musicatradicional.eu. La base de datos se ha desarrollado recientemente en el marco del proyecto «Polifonía hispana y música de tradición oral en la era de las humanidades digitales» (HAR2016-75371-P), durante el período 2017-2020. 
complementariedad entre los diversos medios utilizados por los folcloristas a mediados del siglo xx para realizar su trabajo de campo -principalmente transcripciones manuscritas en el caso de las misiones folclóricas y concursos del FMT y grabaciones sonoras y fotografías en la colección de Lomax-. De esta forma, se produce un enriquecimiento mutuo entre colecciones a través de la tecnología. La base de datos me permitió estudiar la colección española de Alan Lomax e interpretar la información de modo más contextualizado y sirvió como punto de partida para crear una aplicación basada en la tecnología de sistemas de información geográfica que presenta el contenido de la base de datos de forma más visual e intuitiva. De este modo, como se explicará a continuación, ambos recursos digitales ponen a disposición de los investigadores una catalogación de todos los documentos tanto escritos como sonoros de la colección de música española de Lomax, y permiten a la ciudadanía en general consultar y disfrutar de la historia oral de pequeñas localidades de la España de mediados del siglo xx.

\section{LA DISPONIBILIDAD DE LA COLECCIÓN DE MÚSICA ESPAÑOLA DE ALAN LOMAX EN LA BASE DE DATOS DEL FONDO DE MÚSICA TRADICIONAL DEL IMF-CSIC}

La colección de música española de Alan Lomax se ha catalogado en la base de datos del FMT con la signatura «Z1952-1953 LOMAX». ${ }^{13}$ Este trabajo supuso la incorporación de la totalidad de las grabaciones realizadas por el folclorista en su viaje por España, incluyendo 1.480 piezas musicales, 100 localidades, 665 colaboradores y 255 unidades documentales que contienen miles de documentos escritos (tales como cartas, cuadernos de viaje, diarios y mapas anotados, entre otras tipologías) de la Library of Congress. Como el resto de las fichas básicas de cada una de las «fuentes» o colecciones incorporadas a esta base de datos, la ficha catalográfica general de la colección de Lomax incluye la fecha, el nombre del investigador, una descripción física del material, el número de piezas que componen la colección y listados de todas las localidades representadas (donde se grabaron las piezas o de las que procedían los intérpretes de las mismas), las piezas catalogadas, las personas que participaron cantando o tocando, los documentos escritos resultado del trabajo de campo, y bibliografía relacionada con la colección (Figura 1). Se ha completado una ficha básica de Alan Lomax y otra de su asistente Jeantte Bell, como los investigadores vinculados a esta colección, con los siguientes datos: nombre, fecha de nacimiento y defunción, biografía, referencias y fotografías (Figura 2). También se han creado fichas de otros investigadores que contribuyeron en diferentes grados y de diversas maneras a la colección de música española de Lomax, como Eduardo Martínez Torner, el antropólogo Julio Caro Baroja (1914-1995) o el compositor y colector de música folclórica Patrick Shuldham-Shaw (1917-1977), entre otros.

Las localidades visitadas por Lomax, los datos de las personas que cantaron y tocaron para él y los detalles de las piezas que grabó se han catalogado en la base de datos del FMT utilizando como punto de partida las grabaciones disponibles

13 A. Mazuela-Anguita, «Z1952-1953 LOMAX», Fondo de Música Tradicional IMF-CSIC, ed. E. RosFábregas, https:/ / musicatradicional.eu/Lomax. 
en línea en el archivo de la Association for Cultural Equity, una asociación basada en el Hunter College de Nueva York y fundada por el propio Lomax en 1983 con la finalidad de preservar e investigar las tradiciones musicales populares a nivel mundial. ${ }^{14}$ Además de los detalles disponibles en el archivo de la Association for Cultural Equity, la audición en detalle de la totalidad de las grabaciones ha permitido, en un segundo estadio, corregir o matizar la información y añadir datos como el íncipit textual de las canciones (Figuras 3 y 4). El análisis de las cajas originales de las cintas conservadas en el American Folklife Center de la Library of Congress ha resultado fundamental para completar la catalogación de piezas, lugares e intérpretes, clarificar aspectos del itinerario de Lomax en España, determinar fechas de grabación confusas e identificar a intérpretes. En las cajas de las cintas Lomax realizaba anotaciones durante las sesiones de grabación -que continuaba en los cuadernos de campo- y también posteriormente en Londres cuando revisaba las grabaciones junto al musicólogo Eduardo Martínez Torner. Por ejemplo, marcaba con dos asteriscos las piezas que consideraba aptas para su publicación discográfica.

La información que proporcionan las grabaciones y las cajas de las cintas se ha completado con datos extraídos de los cuadernos de campo y otra documentación en papel de la colección de Lomax. Se ha llevado a cabo un estudio históricocrítico y filológico de fuentes escritas como cartas, fragmentos de cuadernos de campo y diarios de viaje que, como valioso complemento de las fuentes sonoras, se han analizado y editado. Por ejemplo, se han examinado, transcrito y traducido al español todos los diarios y escritos de Lomax vinculados con su trabajo en España, ordenándolos cronológicamente siguiendo su itinerario por el país, y se han catalogado en la base de datos, relacionándolos con las personas, piezas y lugares mencionados (Figura 5). Los diarios y cuadernos de campo constituyen tipos documentales que se complementan entre sí. Los diarios consisten principalmente en hojas mecanografiadas y presentan un estilo más elaborado que el de los cuadernos de campo, preservados en notas manuscritas y que son en ocasiones intensamente personales. Cada uno de los documentos catalogados en la base de datos contiene un enlace que remite a su digitalización, cuando está disponible, en la colección digital de la Library of Congress. ${ }^{15}$

De este modo, la base de datos permite, por ejemplo, buscar el nombre de un determinado informante, obtener sus datos, una lista de las piezas que interpretó para Lomax, qué comentarios hizo el folclorista sobre el informante y su interpretación musical, y una lista de la documentación en la que se le menciona -como correspondencia, cuadernos de campo y diarios, entre otros tipos documentales- $-{ }^{16}$ También se han analizado, transcrito y traducido los comentarios de Lomax sobre las localidades españolas que visitó y estos comentarios se han incorporado a la base de datos. Se han escuchado los programas de radio que

14 Esta asociación gestiona los derechos de propiedad intelectual de muchas de las grabaciones de Alan Lomax; véase https:/ / archive.culturalequity.org.

15 https:/ / www.loc.gov/collections/alan-lomax-manuscripts/about-this-collection.

16 En la base de datos se utiliza el término «informante» para denominar a las personas que cantaron o tocaron las piezas recogidas por los folcloristas. Aunque es un término ampliamente utilizado en la disciplina antropológica, ha sido cuestionado y actualmente tiende a sustituirse por otros como «colaborador», con el propósito de reflejar la importancia de estas personas en el proceso y descentralizar la figura del etnomusicólogo. Véanse, por ejemplo, RichardSON (1975); MetCALf (2002: 43); y MORTENSEN (2005). 
realizó Lomax para la BBC sobre su viaje por España y se han analizado los scripts y guiones en borrador de los mismos, incorporando a la base de datos los comentarios relativos a personas, piezas musicales y lugares específicos. Asimismo, se han estudiado las publicaciones discográficas realizadas por Lomax de las grabaciones españolas y las editadas posteriormente por los investigadores, indicando en la ficha de cada pieza en la base de datos si ha sido publicada e incorporando los comentarios que sobre la misma realizaron los editores de los discos. ${ }^{17}$

El archivo online de la Association for Cultural Equity incluye digitalizaciones de las fotografías realizadas por Lomax en España. De este modo, ha sido posible incorporar a la ficha de algunos intérpretes y colaboradores fotografías tomadas por Lomax, remitiendo al archivo de la Association for Cultura Equity. En bastantes casos, las personas que aparecen en las fotografías no habían sido identificadas. Gracias al estudio comparativo de la colección documental completa ha sido posible llevar a cabo numerosas identificaciones. Además, en uno de sus cuadernos de campo, correspondiente al trabajo realizado en Mallorca y Valencia, Lomax hace una lista de lo incluido en algunos de los carretes fotográficos. ${ }^{18}$ Por ejemplo, es posible identificar al músico y etnomusicólogo chileno Pablo Garrido (1905-1982) en algunas de las fotografías de Lomax (Figura 6); gracias a los cuadernos de campo, los diarios de Bell y las cartas que se intercambiaron Lomax y Garrido, podemos determinar que ambos coincidieron en el festival de folclore de Palma en junio de 1952, y que en agosto Garrido alojaría a Lomax y Bell en su hacienda cercana a Tabernes de Valldigna (Valencia) y los acompañaría a una sesión de grabación con la banda de música de Villanueva de Castellón.

17 Algunas de las grabaciones de música española de Lomax fueron publicadas por primera vez en 1955 como parte de la World Library of Folk and Primitive Music, la colección de LP de Columbia (World Library of Folk and Primitive Music, vol. 13: Spain, LP, Columbia, 1955), que se reeditaría en CD en 1999 por la discográfica Rounder (World Library of Folk and Primitive Music, vol. 4: Spain, ed. Josep Martí, CD, Rounder, 1999). Después de 1958, Westminster publicó la colección Songs and Dances of Spain (Songs and Dances of Spain, 11 LP, Westminster, [después de 1958]. Judith R. Cohen se encargó de coordinar la colección de CD publicada por Rounder entre 2001 y 2006 (The Alan Lomax Collection. The Spanish Recordings, ed. Judith R. Cohen, 6 CD, Rounder, 2001-2006). Sobre el trabajo en este proyecto discográfico, véase Cohen, Berlanga, Costa y Torres, 2004. Cohen también editó en 2011 un doble CD con grabaciones asturianas (Alan Lomax in Asturias, ed. Judith R. Cohen, 2 CD, Global Jukebox Media, 2011). Radio Clásica de Radio Nacional de España ha dedicado desde 2011 varios programas a las grabaciones de Alan Lomax en España, en el contexto de espacios como Músicas de tradición oral y La Riproposta. En Músicas de tradición oral Gonzalo Pérez Trascasa dedicó varios programas a Lomax: «Alan Lomax en España» (emitido el 11 de junio de 2011), «Alan Lomax en Asturias» (5 de noviembre de 2011), «Alan Lomax en el País Vasco» (29 de enero de 2014), «Grabaciones extremeñas de Alan Lomax» (12 de marzo de 2014) y «Otras grabaciones españolas de Alan Lomax» (9 de diciembre de 2015). Yolanda Criado en La Riproposta, presentó el programa «Los viajes de Alan Lomax por España» (28 de octubre de 2017). En el ámbito audiovisual, en 2007 se publicó el documental Lomax: The Songhunter, escrito y dirigido por Rogier Kappers, que se había emitido en televisión (en la serie «Point of View» de PBS) el año anterior, y que consiste en un viaje por Europa realizado en 2004 con parada en España, buscando a las personas que habían interpretado música para Lomax cincuenta años antes; véase Lomax: The Songhunter, dir. Rogier Kappers, DVD, Rounder, 2007. Un proyecto similar se ha realizado más recientemente y ha supuesto la emisión en La 2 (RTVE) de tres programas de la Serie Música de la Universidad Nacional de Educación a Distancia (UNED) titulados Tras los pasos de Alan Lomax en las localidades segovianas de Vegas de Matute (emitido el 25 de noviembre de 2016) y Zarzuela del Monte (10 de febrero de 2017) y en la localidad toledana de Lagartera (7 de julio de 2017). 18 Cuaderno de campo de Alan Lomax en Mallorca y Valencia, AFC 2004/004: MS 03.02.13. 
Se ha analizado y transcrito la totalidad de la correspondencia de Lomax relacionada con su trabajo en España. Se trata de centenares de cartas, la mayor parte dirigidas a los intérpretes y colaboradores sobre asuntos relacionados con la autorización para el uso de las grabaciones en programas de radio y publicaciones discográficas. También encontramos cartas de agradecimiento a personas que lo ayudaron en algún momento del viaje, lo que permite analizar el modo en que se configuró la red de contactos de Lomax en España y cómo esta red determinó o no su itinerario, las personas a las que grabó y la música que compiló (MAzUELAANGUITA, 2021b). Estas cartas, que se conservan generalmente en dos versiones -una en inglés y otra en español, seguramente traducidas por un asistente- han resultado fundamentales también para clarificar la identidad de intérpretes no identificados en la catalogación de las grabaciones disponible en el archivo de la Association for Cultural Equity. La transcripción completa de estas cartas se ha incorporado a la base de datos, conectando cada documento con personas, lugares y piezas (Figura 7). La correspondencia refleja, además, qué piezas musicales de las compiladas en España eran las mejor consideradas por Lomax. Por tanto, a través de esta base de datos en línea se ha puesto a disposición de los investigadores la información completa de la colección de música española de Alan Lomax de forma interrelacionada y en un formato que permite localizar y acceder fácilmente a datos concretos, grabaciones sonoras, transcripciones de documentos escritos en formato buscable, y digitalizaciones de documentación original.

\section{CARTOGRAFÍA SONORA DEL ITINERARIO DE ALAN LOMAX A TRAVÉS DE LA APLICACIÓN «ALAN LOMAX'S JOURNEY ACROSS SPAIN (1952-53)»}

Gracias a su procesamiento a través de la base de datos del FMT, el análisis comparativo de la gran variedad de documentos conservados en el American Folklife Center de la Library of Congress que conforman los resultados materiales del viaje de Alan Lomax por España también ha permitido dilucidar el itinerario que siguió el etnomusicólogo en su periplo por el país, el cual difiere en ocasiones del establecido en estudios previos sobre el trabajo de Alan Lomax en España. ${ }^{19}$ La documentación contable ha resultado de especial utilidad para clarificar el itinerario y fechas del viaje, puesto que las anotaciones en las cajas de las cintas, los cuadernos de campo y los diarios ofrecen en numerosos casos datos contradictorios. En la colección Lomax se conservan facturas de alojamiento, restaurantes y talleres mecánicos donde reparó su coche, entre otra documentación de tipo económico que proporciona fechas concretas. Asimismo, se han analizado los mapas de España que utilizó Lomax, algunos de ellos con anotaciones y marcas.

Lomax llegó al festival de Palma en junio de 1952 desde París, conduciendo su propio coche desde París hasta Barcelona, desde donde zarpó hacia Mallorca. Tras la finalización del festival, pasó más de un mes haciendo trabajo en las islas

19 Véanse, por ejemplo, los comentarios incluidos en la catalogación de las grabaciones de Lomax en el archivo de la Association for Cultural Equity (https:/ / archive.culturalequity.org), así como las notas de The Alan Lomax Collection. The Spanish Recordings, ed. Judith R. Cohen, 6 CD, Rounder, 2001-2006. 
Baleares y, a inicios de agosto, volvió a Barcelona donde se reunió con Jeanette Bell, que acababa de llegar de Londres y participaría en el proyecto a partir de entonces como su asistente. Juntos emprenderían un viaje en coche hacia Valencia donde grabaron en varias localidades y después se dirigirían a Madrid, que funcionó como un tipo de base central organizar el trabajo, establecer contactos y planificar los siguientes pasos. Desde Madrid viajaron al País Vasco y, pasando de nuevo por Madrid, se dirigieron a Toledo. Las siguientes grabaciones se hicieron en Castilla-La Mancha (entonces región de Castilla la Nueva) a inicios de septiembre y después pasaron a Andalucía, comenzando por Granada y yendo hacia el oeste. Prosiguieron su trabajo de campo en Extremadura y, tras pasar unos días en Madrid, visitaron Aragón. Volvieron a Madrid, desde donde hicieron un viaje de ida y vuelta a Segovia y grabaron en localidades de la provincia. Después partieron hacia León y grabaron en algunas de los pueblos donde sobrevivía la cultura maragata. Las siguientes áreas que visitaron fueron Asturias, Cantabria y Galicia. Volvieron a Madrid y desde allí realizaron un viaje de ida y vuelta a Murcia, pasaron la Noche Buena en Largartera (Toledo) y, en el último tramo del trayecto, se dirigieron al norte de nuevo, haciendo un alto en Yebra de Basa, una remota localidad en las montañas de Huesca. Llevaron a cabo sus últimas grabaciones en Navarra y el País Vasco en los primeros días de enero de 1953.

Se ha creado una aplicación online de libre acceso basada en un mapa interactivo que contiene este itinerario de Lomax, con el objetivo de dar a conocer los resultados sonoros, visuales y escritos del viaje por España al público general (Figura 8) ${ }^{20}$ Durante mi estancia como «postdoctoral research fellow» en la Library of Congress, la Digital Initiatives Division de la biblioteca me proporcionó acceso institucional a ArcGIS, lo que me permitió explorar las herramientas que proporcionan los sistemas de información geográfica online y aplicar lo aprendido al proyecto de análisis de la configuración de la colección de música española de Alan Lomax. Opté por crear un StoryMap, que permite «narrar» historias -en este caso el viaje de Lomax por España- mediante la combinación de contenido multimedia con la cartografía digital. En un primer estadio de mi trabajo con ArcGIS, georreferencié el mapa de carreteras original de 1952 utilizado Lomax y conservado en el American Folklife Center, ${ }^{21}$ y utilicé este mapa georreferenciado para generar una primera versión del proyecto de StoryMap. Sin embargo, finalmente opté por mostrar en la aplicación únicamente un mapa global actual que simplificara la navegación por parte del usuario.

Para cada punto del recorrido, se incluyó una imagen, un ejemplo sonoro, una breve descripción y un enlace a la base de datos del FMT correspondiente a esa localidad, donde se encuentra la información completa de la música recopilada tanto por Lomax como por otros investigadores en el lugar, las personas que participaron como intérpretes y la documentación de la colección Lomax relacionada específicamente con la localidad. La Figura 9 presenta, como ejemplo, una captura del contenido multimedia correspondiente a la localidad leonesa de Lumajo, consistente en la grabación de «Salgan las de la cocina», una canción sobre las fiestas de boda en la región, interpretada por un grupo

20 A. Mazuela-Anguita, «Alan Lomax's journey across Spain (1952-53)», aplicación online (StoryMap) de libre acceso, http://arcg.is/2x1K6u0.

21 Mapa Firestone-Hispania (c. 1952), anotado por Lomax, AFC 2004/004: MS 03.03.27. A. MazuelaAnguita, «AFC 2004/004: MS 03.03.27», Fondo de Música Tradicional IMF-CSIC, ed. E. Ros-Fábregas, https://musicatradicional.eu/document/30023. 
de mujeres que cantaban y tañían panderos cuadrados y grabada por Lomax el 6 de noviembre de 1952; una fotografía tomada por Lomax de una mujer de la localidad llamada Emilia del Ama sosteniendo un pandero cuadrado; y un enlace a la entrada "Lumajo» en la base de datos del FMT. ${ }^{22} \mathrm{Al}$ acceder a la base de datos a través de ese enlace es posible navegar por listados de piezas recogidas en Lumajo, tanto por Lomax como por otros folcloristas, así como encontrar listas de habitantes que aportaron piezas musicales a los recopiladores, otras fotografías tomadas por Lomax en el lugar, y transcripciones y digitalizaciones de documentos (correspondencia, cuadernos de campo, diarios) resultantes del trabajo de campo de Lomax en lo que él consideraba en sus cuadernos de campo el «pueblo más básicamente vaqueiro de todos». ${ }^{23}$

Esta aplicación, que fue presentada en un congreso organizado por la Digital Initiatives Division de la Library of Congress en julio de 2017, ${ }^{24}$ intenta hacer los datos más visuales y accesibles no solo a investigadores sino también a una audiencia más amplia fuera del ámbito puramente académico. Fue seleccionada como ejemplo de «digital scholarship» por la Digital Initiatives Division de la Library of Congress. ${ }^{25}$

\section{CONCLUSIONES}

Alan Lomax creó una de las primeras colecciones sistemáticas de música tradicional española, ${ }^{26}$ y los resultados sonoros, visuales y escritos de su viaje reflejan, quizás solo en parte, el enorme impacto que debió tener su visita entre los habitantes de las pequeñas localidades de la España franquista. Los resultados del estudio de esta colección a través de la tecnología y usando la historia oral como marco conceptual permiten constatar que el fascinante legado de Lomax no solo tiene un enorme valor antropológico y musical en sí mismo, sino que también complementa y enriquece otras colecciones de música de tradición oral recopiladas en España en los mismos años por parte de folcloristas españoles. El procesamiento de la colección de música española de Alan Lomax en forma de «datos» y las relaciones establecidas entre estos me han permitido analizar, comprar e interpretar la información de forma más contextualizada y poner a

\footnotetext{
22 A. Mazuela-Anguita, «Salgan las de la cocina (Lumajo, León, 11/1952)», Fondo de Música Tradicional IMF-CSIC, ed. E. Ros-Fábregas, https://musicatradicional.eu/piece/29359; y «Ama, Emilia del», https://musicatradicional.eu/informant/28770.

23 Cuaderno de campo de Alan Lomax en Asturias y León, AFC 2004/004: MS 03.02.31. A. MazuelaAnguita, «AFC 2004/004: MS 03.02.31», Fondo de Música Tradicional IMF-CSIC, ed. E. Ros-Fábregas, https://musicatradicional.eu/document/30436.

24 A. Mazuela-Anguita, «Mapping Inter-Institutional Connections Through Digital Humanities: The Alan Lomax Collection of Spanish Folk Music (1952-53) at the Library of Congress», «lighting talk» en el congreso internacional Collections as Data: IMPACT, Washington D. C., Library of Congress, 25 de julio de 2017.

25 https://labs.loc.gov.

26 Las primeras grabaciones sistemáticas de campo de música folclórica española, realizadas en discos de aluminio entre julio de 1932 y enero de 1933 -justo veinte años antes que las grabaciones de Lomax - por Kurt Schindler (1882-1935), musicólogo alemán nacionalizado estadounidense. Fueron promovidas por el Centro de Estudios Históricos de Madrid, dirigido por Ramón Menéndez Pidal; véase SCHINDLER, 1941.
} 
disposición de los investigadores y de la ciudadanía en general este fascinante patrimonio musical, conservado en forma de documentos sonoros, visuales y escritos íntimamente conectados entre sí. Lo hace, además, de una forma interactiva, permitiendo búsquedas de datos específicos y la interrelación de estos.

A través de la base de datos y la aplicación cartográfica que se han presentado en este artículo, es posible que descendientes de las personas que cantaron y tocaron para Lomax en 1952 y 1953 puedan recuperar la memoria musical de sus familias, que los habitantes de las pequeñas localidades donde el folclorista llevó a cabo trabajo de campo puedan conocer su legado musical local, que el profesorado de escuelas, institutos de enseñanza secundaria y bachillerato y conservatorios puedan usar estas melodías con fines y aplicaciones didácticas, que los intérpretes actuales de músicas folclóricas tengan a su disposición un ingente repertorio que recrear, y que la comunidad en general pueda disfrutar de esta magnífica colección. De este trabajo se desprende la importancia de la tecnología digital no solo como un medio para habilitar la difusión instantánea de los hallazgos científicos y promover el intercambio académico, sino también como una herramienta para repensar la investigación humanística y acercarla a la comunidad.

\section{DISCOGRAFÍA}

World Library of Folk and Primitive Music, vol. 13: Spain, LP, Columbia, 1955.

Songs and Dances of Spain, 11 LPs, Westminster, [después de 1958].

World Library of Folk and Primitive Music, vol. 4: Spain, ed. J. Martí, CD, Rounder, 1999.

The Alan Lomax Collection. The Spanish Recordings, ed. J.R. Cohen, 6 CD, Rounder, 2001-2006.

Lomax: The Songhunter, dir. R. Kappers, DVD, Rounder, 2007.

Alan Lomax in Asturias, ed. Judith R. Cohen, 2 CD, Global Jukebox Media, 2011.

\section{RECURSOS WEB}

Association for Cultural Equity, https:/ / archive.culturalequity.org.

Library of Congress, Alan Lomax Collection, https://www.loc.gov/collections/ alan-lomax-manuscripts/about-this-collection.

Mazuela-Anguita, A. «Alan Lomax's journey across Spain (1952-53)», aplicación online (StoryMap) de libre acceso, http:/ / arcg.is/2x1K6u0.

Mazuela-Anguita, A. "Z1952-1953 LOMAX», Fondo de Música Tradicional IMFCSIC, ed. E. Ros-Fábregas, https:/ / musicatradicional.eu/Lomax.

Ros-FÁbregas, E. (Ed.): Fondo de Música Tradicional del CSIC (1944-1960), Barcelona, Consejo Superior de Investigaciones Científicas, Institució Milà i Fontanals, https:/ / musicatradicional.eu. 


\section{REFERENCIAS}

BARON, R. (2012): «"All Power to the Periphery": The Public Folklore Thought of Alan Lomax», Journal of Folklore Research, 49 (3): 275-317.

CALvo, L. (1989): «La Etnomusicología en el Instituto Español de Musicología», Anuario Musical, 44: 167-197.

CAlvo, L. (1989-1990): «Higini Anglès y la "Obra del Cançoner Popular de Catalunya"», Recerca Musicològica, 9-10: 283-293.

CAsado Lobato, C. (2003): «Alan Lomax en Maragatería», Argutorio, 5 (11): 33.

Casado Lobato, C.; Porro Fernández, C.A. (2011): «Los registros sonoros de Alan Lomax en Castrillo de los Polvazares y Villalibre de Somoza (León) en 1952 (III)», Revista de Folklore, 358: 27-38.

Casado Lobato, C.; Porro Fernández, C.A. (2012): «Los registros sonoros de Alan Lomax en El Val de San Lorenzo (León) en 1952 (y IV)», Revista de Folklore, 362: 23-35.

CoHen, J. R. (2002): «Spain: Lomax Remembered», Canadian Folk Music, 36 (4): 22.

CoHen, J. R. (2011a): «lbiza and Formentera: Worlds of Singers and Songs», en G. Baldacchino (ed.), Island Songs: A Global Repertoire, The Scarecrow Press, Londres, Toronto y Plymouth: 223-245.

CoHEN, J. R. (2011b): «Sobre las grabaciones de Alan Lomax en España en 1952. Los escritos de Alan Lomax traducidos por Judith R. Cohen», en J. R. CoHEN (ed.), Alan Lomax in Asturias, 2 CD, Global Jukebox Media: 157-167.

Cohen, J. R.; Berlanga, M.A.; Costa, L.; Torres, J.A. (2004): «Alan Lomax en España», en J. Martí y S. MartíneZ (eds.), Voces e imagenes en la etnomusicologia actual, Museo del Traje, Madrid: 399-410.

DAVIS, M.E. (2015): «Oral History, Musical Biography, and Historical Ethnomusicology», en V. L. LeVINE y P. V. Bohlman (eds.), This Thing Called Music. Essays in Honor of Bruno Nettl [Europea: Ethnomusicologies and Modernities 18], Rowman \& Littlefield, Lanham, Boulder, New YorkLondon: 255-266.

Gembero-Ustárroz, M. (2011): «Músicas de tradición oral en Navarra (1944-1947): recopilaciones conservadas en la Institución Milá y Fontanals del CSIC en Barcelona», Príncipe de Viana, 72 (253): 411-462.

Gembero-Ustárroz, M.; Ros-Fábregas, E. (eds.) (2021): Musicología en web. Patrimonio musical y Humanidades Digitales [DeMusica 29] Reichenberger, Kassel.

Gregory, E.D. (2002): «Lomax in London: Alan Lomax, the BBC and the Folk-Song Revival in England, 1950-1958», Folk Music Journal, 8 (2): 136-169.

LomaX, A. (1960): «Saga of a Folksong Hunter», Hi-Fi Stereo Review, 4 (5): 38-46.

Lomax, A. (2003): Selected Writings 1934-1997, ed. de R.D. Cohen, con ensayos introductorios de G. AveriLl et al., Routledge, Nueva York y Londres.

Lomax, A. (2011): Alan Lomax, Assistant in Charge. The Library of Congress Letters, 1935-1945, ed. de R.D. CoHen, University Press of Mississippi, Jackson.

MARTí, J. (1997): «Folk Music Studies and Ethnomusicology in Spain», Yearbook for Traditional Music, 28: 107-140.

Mazuela-Anguita, A. (2015): Las mujeres y la transmisión del repertorio andaluz en el Fondo de Música Tradicional del CSIC-IMF (1945-1960), CIOFF-INAEM, Badajoz.

Mazuela-Anguita, A. (2016): «Identidad cultural y patrimonio audiovisual en 
el Fondo de Música Tradicional del CSIC-IMF (1944-1960)», en V Jornada de Celebración del Día Mundial del Patrimonio Audiovisual. La identidad cultural a través de las colecciones de registros sonoros y audiovisuales. Madrid, 27 de octubre de 2015, Biblioteca Nacional de España, Madrid: 6-9.

Mazuela-Anguita, A. (2021a): «Las grabaciones sonoras de Alan Lomax (19521953) y el Fondo de Música Tradicional IMF-CSIC (1944-1960): vínculos entre colecciones en la era de las humanidades digitales», en M. GEMBERO-UsTÁRROZ y E. Ros-Fábregas (eds.), Musicología en web. Patrimonio musical y Humanidades Digitales [DeMusica 29], Reichenberger, Kassel: 419-452.

Mazuela-Angutta, A. (2021b): Alan Lomax y Jeanette Bell en España (1952-1953): las grabaciones de música folclórica [Colección De Acá y de Allá], Consejo Superior de Investigaciones Científicas, Madrid.

Metcalf, P. (2002): They Lie, We Lie: Getting on with Anthropology, Routledge Falmer, Londres.

Mortensen, C.H. (2005): «(Eco)Mimesis and the Ethics of the Ethnographic Presentation», Journal of American Folklore, 118 (467): 105-120.

PELINSKI, R. (1997): Presencia del pasado en un cancionero castellonense: un reestudio etnomusicológico, Publicacions de la Universitat Jaume I, Castellón.

Perlis, V. (1994): «Oral History and Music», The Journal of American History, 81 (2): 610-619.

PIZÀ, A. (2006): «Una apassionada curiositat visual: Alan Lomax i la seva Leica a Mallorca, Ibiza i Formentera», en A. PIzÀ (ed.), Alan Lomax: mirades: músics $i$ gent de Mallorca, Eivissa i Formentera / miradas: músicos y gente de Mallorca, Ibiza y Formentera / Glances: Musicians and People of Mallorca, Ibiza and Formentera, trad. de J. Mir y D. Bloom, Fundació «Sa Nostra», [Palma]; y Lunwerg, [Barcelona]: 13-35, 133-142.

Porro Fernández, C.A. (2010): «Los registros sonoros de Alan Lomax en Castilla y León: Segovia», Revista de folklore, 346: 111-123.

Porro Fernández, C.A. et al. (2011): «Los registros sonoros de Alan Lomax en Castilla y León: Ribadelago (Zamora), Los Villares de la Reina (Salamanca), Burgos, Lumajo de Laciana y Laguna de Negrillos (León), Octubre de 1952 (II)», Revista de Folklore, 350: 11-25.

RichARDSON, M. (1975): «Anthropologist - The Myth-Teller», American Ethnologist, 2 (3): 517-533.

Ritchie, D.A. (2015 [1995]): Doing Oral History, Oxford University Press, Nueva York.

Schindler, K. (1941): Folk Music and Poetry of Spain and Portugal. Música y poesía popular de España y Portugal, Hispanic Institute in the United States, Nueva York [Una reedición española fue publicada como Música y poesía popular de España y Portugal, ed. facsímil con estudios de I. J. Katz, M. Manzano y S. G. Armistead, Salamanca, Centro de Cultura Tradicional, 1991].

SERrano, P. (dir.) (2000): Aragón visto por Alan Lomax (1952) [Aragón LCD 16], Prames, Zaragoza, [incluye CD].

SzWED, J.F. (2010): Alan Lomax: The Man Who Recorded the World, Viking Penguin, Nueva York.

Vansina, J. (1985): Oral Tradition as History, University of Wisconsin Press, Madison. 


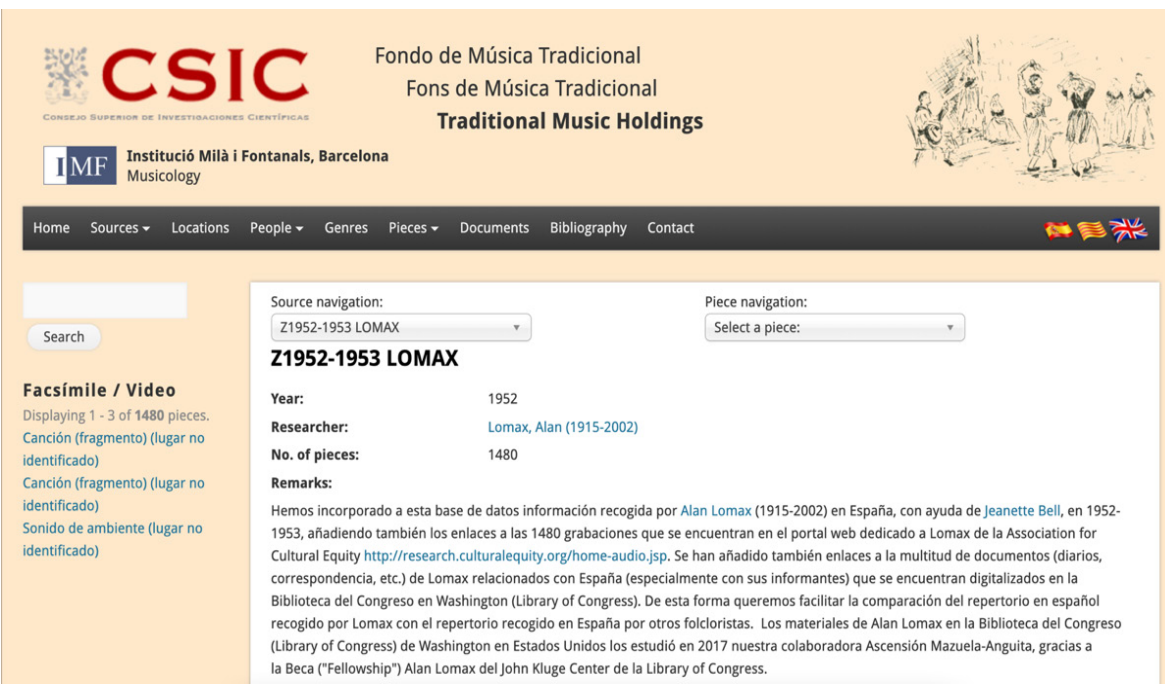

Figura 1. Inicio de la ficha catalográfica general de la colección de música española de Alan Lomax en la base de datos del Fondo de Música Tradicional IMF-CSIC. A. MazuelaAnguita, «Z1952-1953 LOMAX», Fondo de Música Tradicional IMF-CSIC, ed. E. RosFábregas, https://musicatradicional.eu/Lomax.

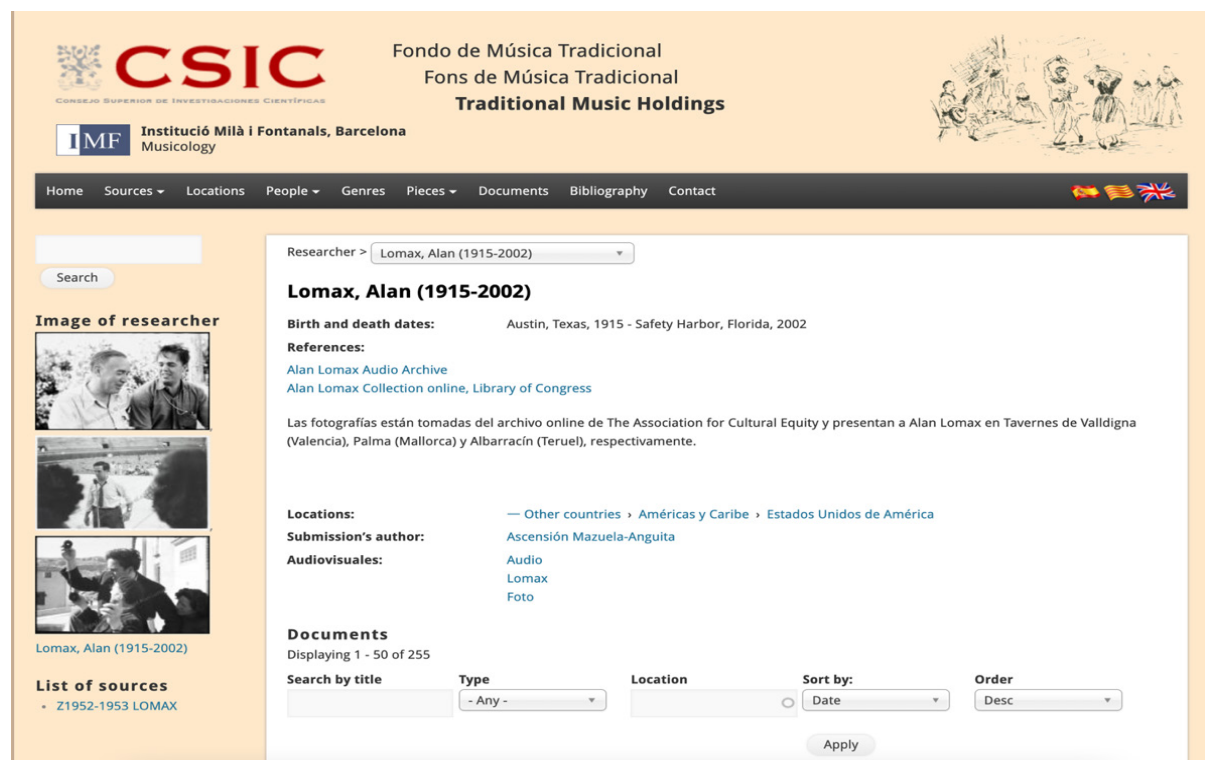

Figura 2. Inicio de la ficha básica de Alan Lomax como investigador en la base de datos del Fondo de Música Tradicional IMF-CSIC. A. Mazuela-Anguita, «Lomax, Alan (1915-2002)», Fondo de Música Tradicional IMF-CSIC, ed. E. Ros-Fábregas, https:/ / musicatradicional.eu/researcher/24038. 


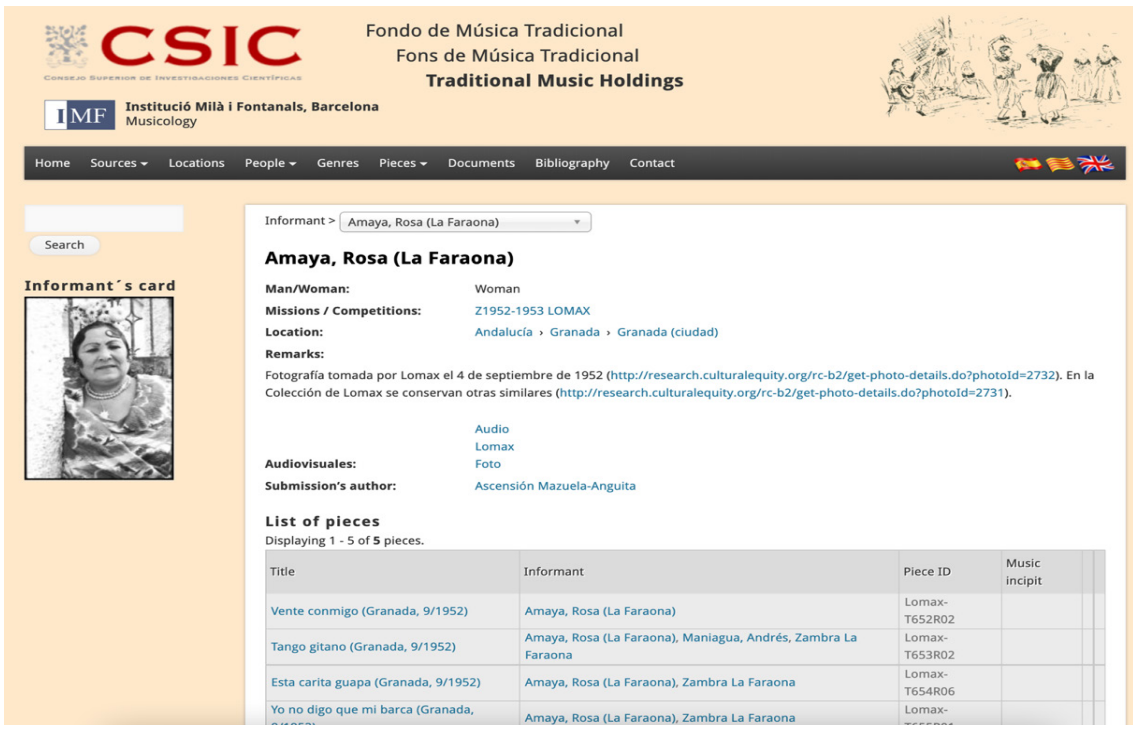

Figura 3. Inicio de la ficha de Rosa Amaya, «La Faraona», que interpretó para Alan Lomax y Jeanette Bell cinco piezas musicales en su zambra del Sacromonte de Granada, en la base de datos del Fondo de Música Tradicional IMF-CSIC. A. Mazuela-Anguita, «Amaya, Rosa (La Faraona)», Fondo de Música Tradicional IMF-CSIC, ed. E. Ros-Fábregas, https://musicatradicional.eu/piece/29966.

Fondo de Música Tradicional
Fons de Música Tradicional
Traditional Music Holdings

Figura 4. Inicio de la ficha de la pieza musical «Vente conmigo» que interpretó Rosa Amaya, «La Faraona», en su zambra del Sacromonte de Granada para Alan Lomax y Jeanette Bell, en la base de datos del Fondo de Música Tradicional IMF-CSIC. A. MazuelaAnguita, «Vente conmigo (Granada, 9/1952)», Fondo de Música Tradicional IMF-CSIC, ed. E. Ros-Fábregas, https://musicatradicional.eu/piece/29966. 


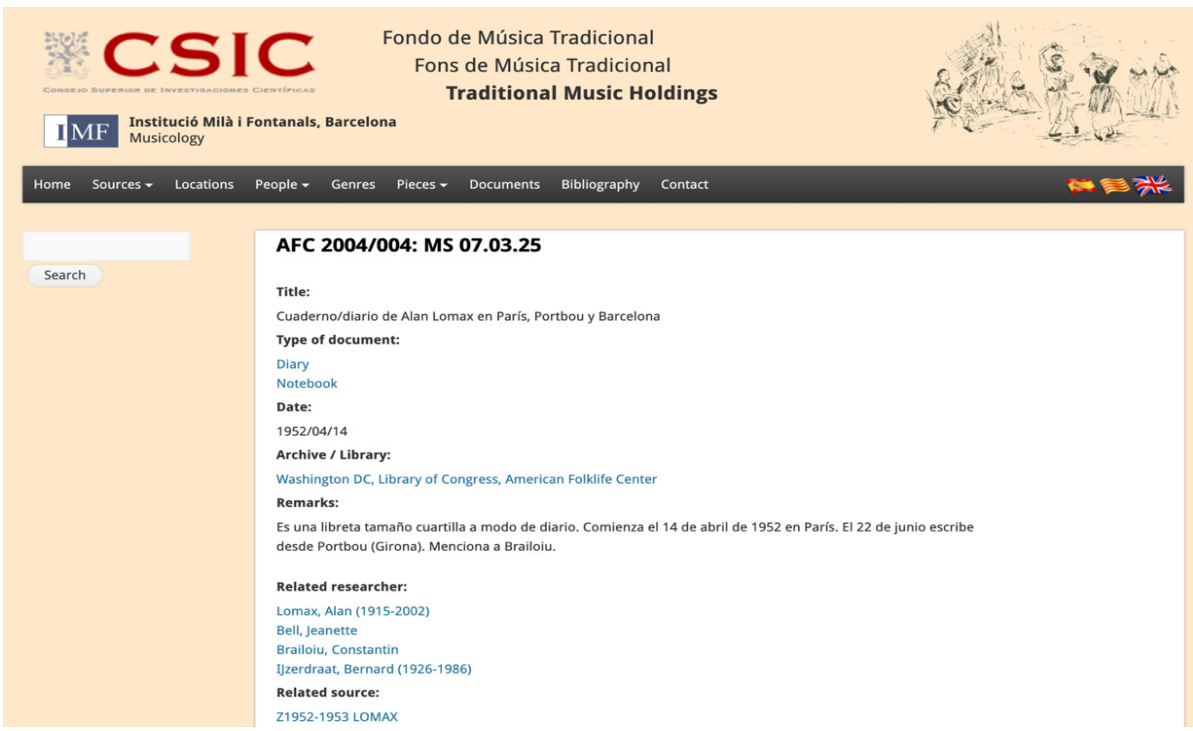

Figura 5. Inicio de la ficha del «Cuaderno/diario de Alan Lomax en París, Portbou y Barcelona», uno de los 255 documentos de la colección de musica española de Alan Lomax incorporados a la base de datos del Fondo de Música Tradicional IMF-CSIC. A. Mazuela-Anguita, «AFC 2004/004: MS 07.03.25», Fondo de Música Tradicional IMF-CSIC, ed. E. Ros-Fábregas, https:// musicatradicional.eu/document/30491.

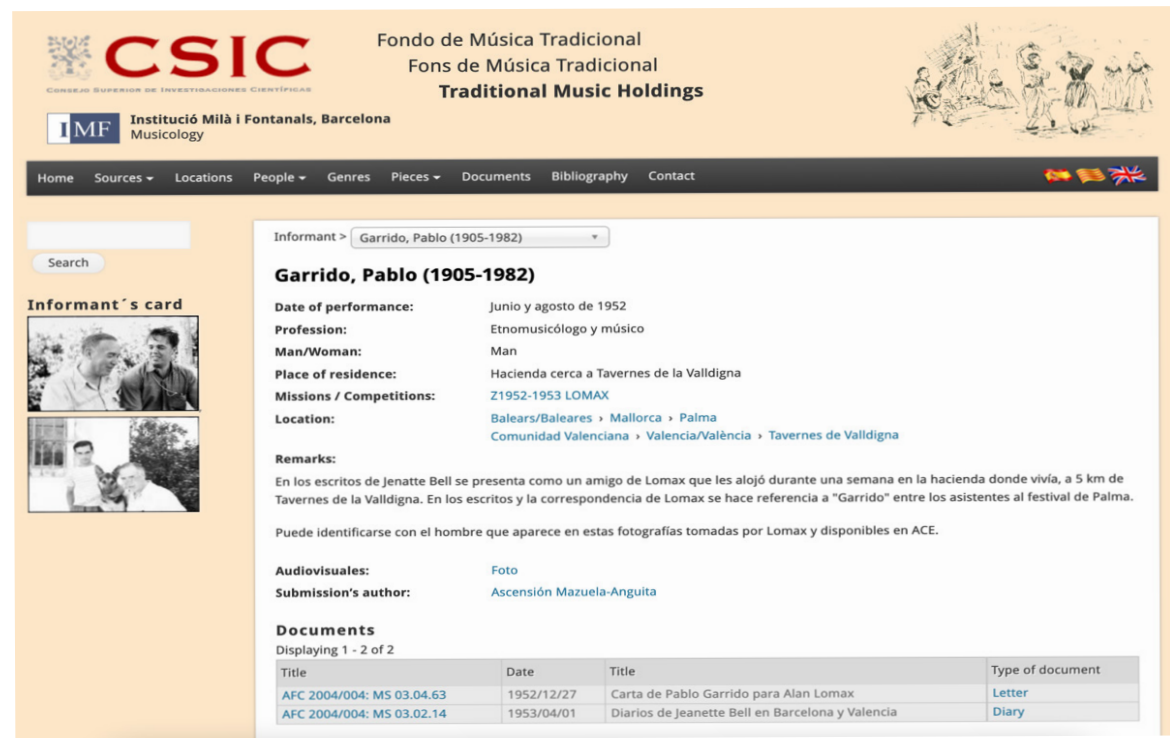

Figura 6. Inicio de la ficha de Pablo Garrido (1905-1982), etnomusicólogo chileno que actuó como uno de los contactos de Alan Lomax en España, en la base de datos del Fondo de Música Tradicional IMF-CSIC. A. Mazuela-Anguita, «Garrido, Pablo (1905-1982)», Fondo de Música Tradicional IMF-CSIC, ed. E. Ros-Fábregas, https://musicatradicional.eu/ informant/30212. 


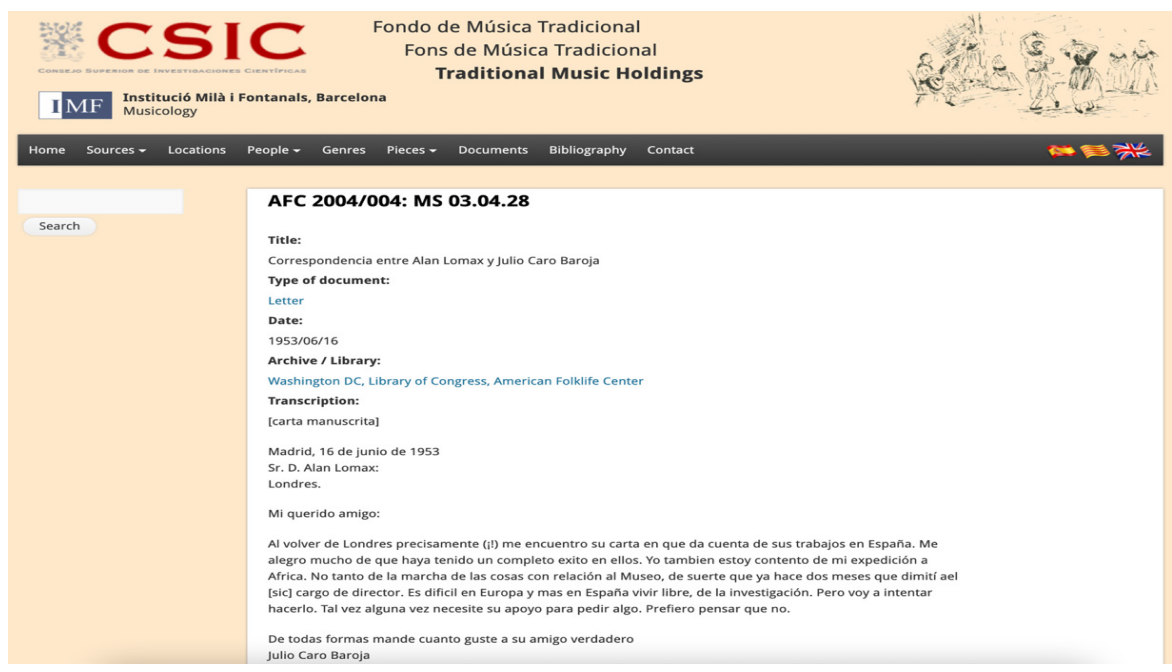

Figura 7. Inicio de la transcripción de la correspondencia completa en Alan Lomax y el antropólogo Julio Caro Baroja (1914-1995) disponible en la base de datos del Fondo de Música Tradicional IMF-CSIC. A. Mazuela-Anguita, «AFC 2004/004: MS 03.04.28». Fondo de Música Tradicional IMF-CSIC, ed. E. Ros-Fábregas, https://musicatradicional.eu/document/30156.

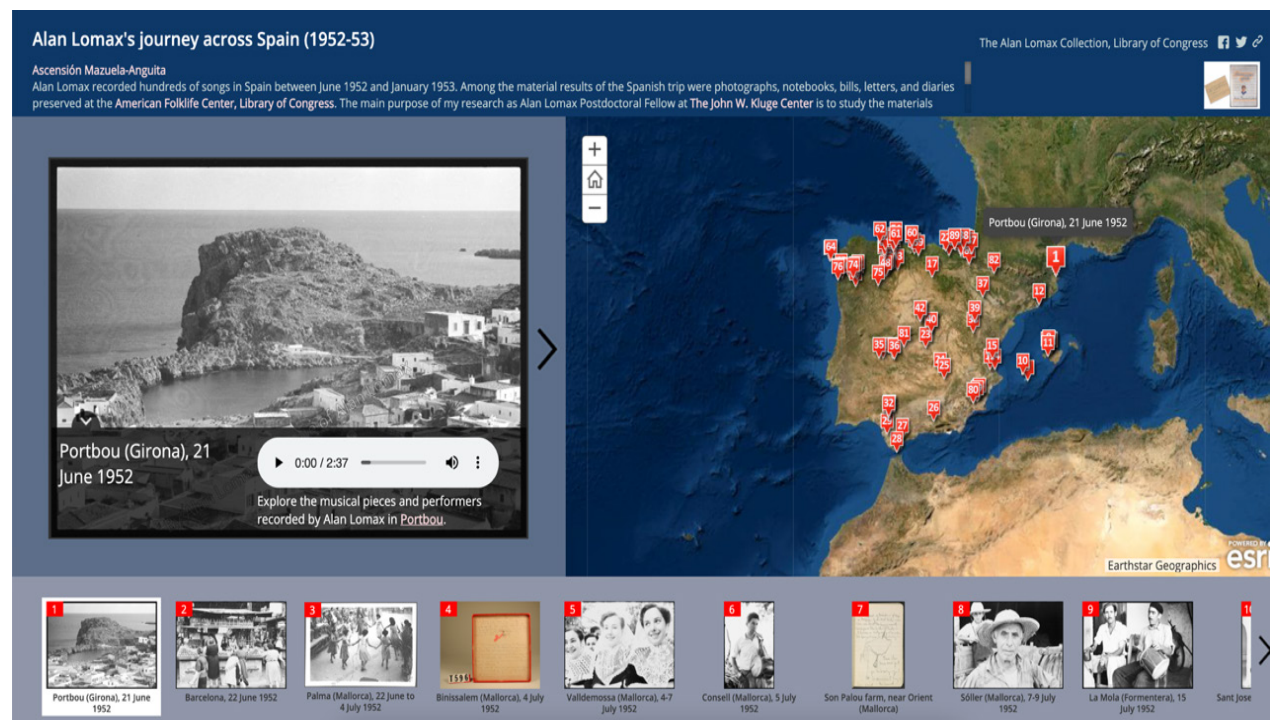

Figura 8. Captura de la interfaz inicial de la aplicación online «Alan Lomax's journey across Spain (1952-53)», http:/ / arcg.is/2x1K6u0. 


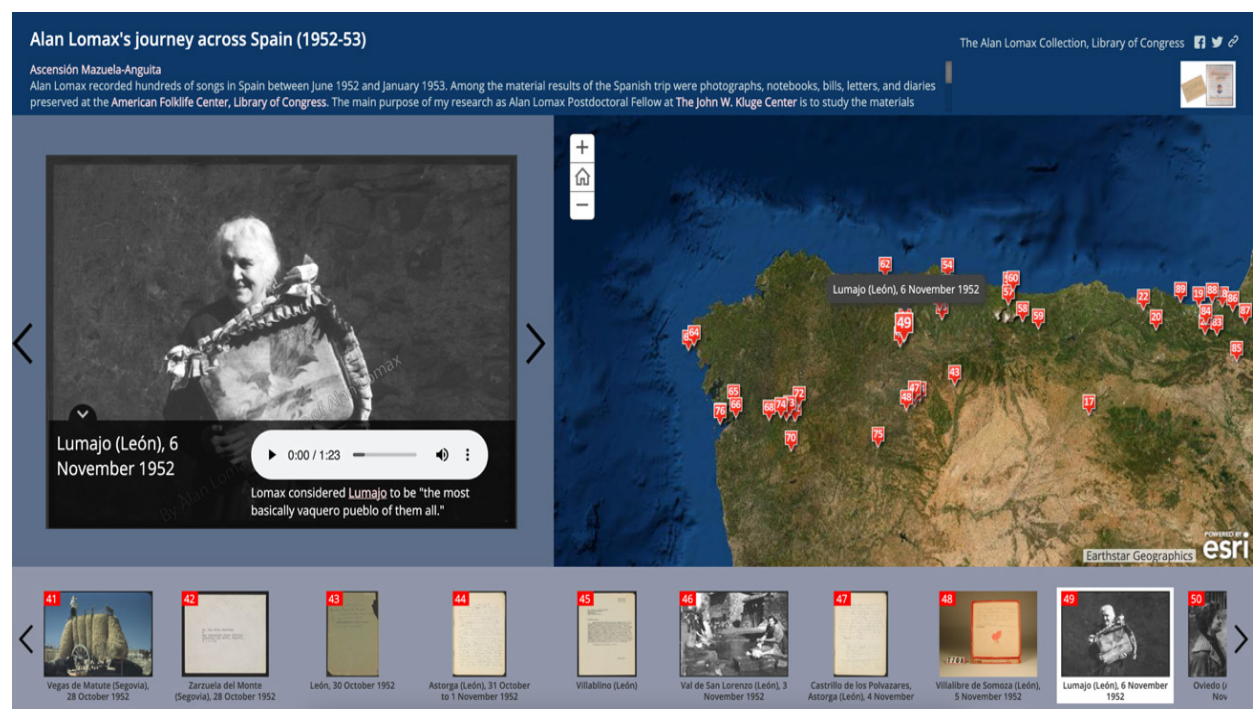

Figura 9. Captura del contenido multimedia correspondiente a la localidad de Lumajo (León) en la aplicación online «Alan Lomax's journey across Spain (1952-53)», http://arcg.is/2x1K6u0. 
